\title{
Un nuevo reto para el comercio exterior peruano: las medidas sanitarias y fitosanitarias
}

\author{
Marcelo Alonso Valverde Arévalo ${ }^{1}$ \\ Universidad Peruana de Ciencias Aplicadas (UPC, Lima, Perú) \\ Ministerio de Comercio Exterior y Turismo del Perú
}

\section{RESUMEN}

El comercio mundial se ha multilateralizado logrando una reducción constante de las medidas arancelarias que lo restringían décadas atrás; sin embargo, los países han encontrado otras medidas que pueden ser utilizadas de manera proteccionista, tales como las medidas sanitarias y fitosanitarias, los obstáculos técnicos al comercio, entre otras. El presente artículo describe y ejemplifica medidas sanitarias y fitosanitarias que han afectado o afectan a las exportaciones peruanas; cómo éstas restringen el comercio y confunden a los exportadores en vez de proteger la salud humana, animal y vegetal.

\section{PALABRAS CLAVE}

Medidas sanitarias y fitosanitarias; comercio exterior; barreras al comercio

\section{A new challenge for Peruvian foreign trade: sanitary and phytosanitary measures}

\footnotetext{
${ }^{1}$ Profesional en negocios internacionales del Vice Ministerio de Comercio Exterior. Miembro del equipo de medidas sanitarias y fitosanitarias. Bachiller en Administración y Negocios Internacionales por la Universidad Peruana de Ciencias Aplicadas (UPC). Correo: mvalverde@mincetur.gob.pe.

Valverde Arévalo, M. A. (2015). Un nuevo reto para el comercio exterior peruano: las medidas santiarias y fitosanitarias. Sinergia e Innovación, 3(1), 1-22.

Fecha de recepción: 07/01/14

Fecha de aceptación: 30/04/15
} 


\section{ABSTRACT}

World trade has become multilateral with a steady reduction of tariff measures that restricted it decades ago; however, countries have found other measures that can be used in a protectionist manner, such as sanitary and phytosanitary measures, technical barriers to trade, among others. This article describes and gives examples of SPS measures that have affected or affect Peruvian exports; how they restrict trade and confuse exporters rather to protect human, animal and plant health.

\section{KEYWORDS}

SPS; foreign trade; barriers to trade 


\section{Antecedentes}

Históricamente los gobernantes se han visto interesados en codificar reglas en materia alimentaria para la proteger la salud de los consumidores contra prácticas que pudiesen resultar fraudulentas (López García \& Berga Monge, 2007). En las tablillas asirias se presentaban descripciones de métodos que se debían aplicar para determinar los pesos y medidas correctos para los cereales que iban a ser destinados al consumo humano. Igualmente, en la antigua Atenas, se inspeccionaban las cervezas y los vinos para determinar su pureza y óptimo estado (López García, \& Berga Monge, 2007).

Del mismo modo durante la Edad Media, en Europa, se aprobaron un número considerable de normativas que versaban sobre la calidad e inocuidad de alimentos tales como huevos, salchichas, quesos, entre otros.

No obstante, al ser cada país libre de establecer sus propias normas sobre inocuidad alimentaria, inevitablemente, se crearon obstáculos al comercio internacional.

Años más tarde, se buscó la manera de estandarizar estas normas por lo que recién en 1961, la FAO crea una entidad encargada de esta labor. El Codex Alimentarius nace con el fin de garantizar la inocuidad de los alimentos para todas las personas y en cualquier parte del mundo. Desafortunadamente, este organismo sólo resolvía una parte de los problemas que afrontaba el comercio.

Las plagas eran un tema aparte para el comercio de alimentos frescos, pues se habían presentado problemas por la propagación de la phylloxera, un áfido norteamericano, que se introdujo de manera accidental en Europa en 1865 y que devastó gran parte de las regiones vitícolas (Convención Internacional de Protección Fitosanitaria, 2014).

Igualmente, las enfermedades de los animales han causado al comercio grandes pérdidas económicas. En el siglo XVIII y a inicios del siglo XIX, la peste bovina ocasionó pérdidas enormes al sector agropecuario por la muerte de alrededor de 200 millones de bovinos (Organización Mundial de Sanidad Animal, 2007, p. 6). Si bien esta enfermedad se llegó a controlar en cierta medida en Europa, en 1920 se reintrodujo accidentalmente en Bélgica por un rebaño de cebúes procedente de las Indias Inglesas destinado a Brasil (Organización Mundial de Sanidad Animal, 2011, p. 5). 
La necesidad de estandarizar normas para la conversación de las plantas y la sanidad animal dieron origen a otras dos instituciones: la Organización Mundial de Sanidad Animal (OIE) y Convención Internacional para la Protección de las Plantas (CIPF).

Estas tres organizaciones pasaron a ser las principales referencias en materia sanitaria y fitosanitaria y el medio para poder controlar el impacto que pudiesen ocasionar al comercio mundial.

\section{Historia del Acuerdo sobre la Aplicación de Medidas Sanitarias y Fitosanitarias}

Posterior a la Segunda Guerra Mundial, existía una gran preocupación por el deterioro de varias economías y la forma de establecer mecanismos para su recuperación.

Una de las soluciones planteadas para resolver este problema fue la propuesta del gobierno de los Estados Unidos conocido como el "Plan Clayton", el cual tenía como objetivo la multilateralización del comercio (Alvarez, s. f.).

Este plan fue discutido en la Conferencia de las Naciones Unidas sobre Comercio y Empleo en la ciudad de La Habana en 1947, logrando la voluntad política de 53 países, por medio de la Carta de La Habana, para el establecimiento de la Organización Internacional de Comercio (OIC). No obstante, el texto de la Carta era provisional con el fin de facilitar las negociaciones en materia arancelaria hasta que se negociara un texto definitivo. En consecuencia se aprobó el Acuerdo General sobre Aranceles Aduaneros y Comercio (GATT) (Alvarez, s.f.).

En el GATT de 1947 no se establecía ninguna estructura jurídica detallada acerca de medidas sanitarias y fitosanitarias. Sin embargo, las medidas nacionales sobre inocuidad de los alimentos, sanidad animal y preservación de las plantas que tenían influencia sobre el comercio estaban sujetas a los artículos I y III y el apartado b) del artículo XX (Organización Mundial del Comercio, 2012, p. 101). Estas normas se aplicaban, por ejemplo, a los límites de residuos de plaguicidas, aditivos alimentarios, restricciones relacionadas con la salud de los animales o la preservación de las plantas.

Posteriormente, en la década de 1970 se propuso la idea de un acuerdo específico sobre los obstáculos técnicos al comercio. Es así que durante la Ronda de Tokio (1973-1979) se negoció el 
Acuerdo Plurilateral sobre Obstáculos Técnicos al Comercio² (o "Código de Normas"). Si bien el objetivo de este acuerdo no era reglamentar las medidas sanitarias y fitosanitarias, se abarcaba prescripciones técnicas ylas resultantes de las medidas relacionadas a la inocuidad alimentaria, la salud humana y animal y la preservación de las plantas (incluidos los límites de residuos de plaguicidas) (Organización Mundial del Comercio, 2012, p. 101).

En la Ronda Uruguay (1985-1994) se consideró que eran insuficientes los derechos y obligaciones en temas sanitarios y fitosanitarios que afectasen al comercio internacional por lo que se negoció un Acuerdo sobre la Aplicación de Medidas Sanitarias y Fitosanitarias en el marco de un Grupo de Trabajo dependiente del Comité de Agricultura (Organización Mundial del Comercio, 2012, p. $101)^{3}$.

Al finalizar esta Ronda, en 1994, se creó la Organización Mundial del Comercio y se adoptó un nuevo acuerdo multilateral: el Acuerdo sobre la Aplicación de Medidas Sanitarias y Fitosanitarias (Acuerdo MSF).

Este acuerdo tiene como objetivo facilitar el comercio de animales, productos y subproductos de origen animal, plantas y productos y subproductos de origen vegetal entre los miembros de OMC, protegiendo al mismo tiempo la vida y salud humana, animal y vegetal. Asimismo, se busca evitar que las medidas sanitarias y fitosanitarias constituyan obstáculos al comercio.

Cabe resaltar que en el acuerdo también se considera necesario fomentar la armonización de normas sobre la base de lo dictado por el CODEX, OIE y CIPF; la transparencia entre las partes; el reconocimiento de zonas libres plagas, entre otros.

\section{Posibles restricciones al comercio}

Los obstáculos al comerciose dividen en términosgenerales entre los derechos de aduana (ad valorem y específicos) y las medidas no arancelarias. Aunque los aranceles aun son el instrumento más utilizado para restringir el comercio, su importancia ha disminuido con la consolidación y el principio de previsibilidad.

\footnotetext{
${ }^{2}$ Un acuerdo es considerado multilateral cuando todos los miembros de OMC se encuentran adscritos a éste y plurilateral cuando sólo algunos miembros forman parte.

${ }^{3}$ lbíd.
}

Valverde Arévalo, M. A. (2015). Un nuevo reto para el comercio exterior peruano: Las medidas sanitarias y fitosanitarias. Sinergia e Innovación, 3(1), 1-22. 
La apertura del comercio ha originado una reducción significativa del nivel medio de los aranceles aplicados. Un ejemplo de este hecho es el arancel medio impuesto por los países desarrollados a todas las importaciones en el período 2010 - 2011 que se situó alrededor del 5\%, mientras que la tasa media aplicada a los productos no agrícolas no superó el 2,5\% (Organización Mundial del Comercio, 2013, p. 55).

En cambio, la aplicación de medidas no arancelarias ha incrementado, tanto por la cantidad de productos afectados como por el número de países que las establecen. Los gobiernos aplican frecuentemente medidas no arancelarias, para conseguir objetivos legítimos de su política de protección de los consumidores frente a enfermedades, pero pueden ser también medidas proteccionistas encubiertas como necesarias para la protección de la salud (Organización Mundial del Comercio, 2013, p. 55-56).

Del total de medidas no arancelarias aplicadas e iniciadas a junio de 2014, los obstáculos técnicos al comercio conjuntamente con las medidas sanitarias y fitosanitarias son las de mayor evolución. En total, las medidas sanitarias y fitosanitarias asciende a 12,792 y cada año van en aumento.

Figura 1 Medidas iniciadas y en vigor al 30/06/2014

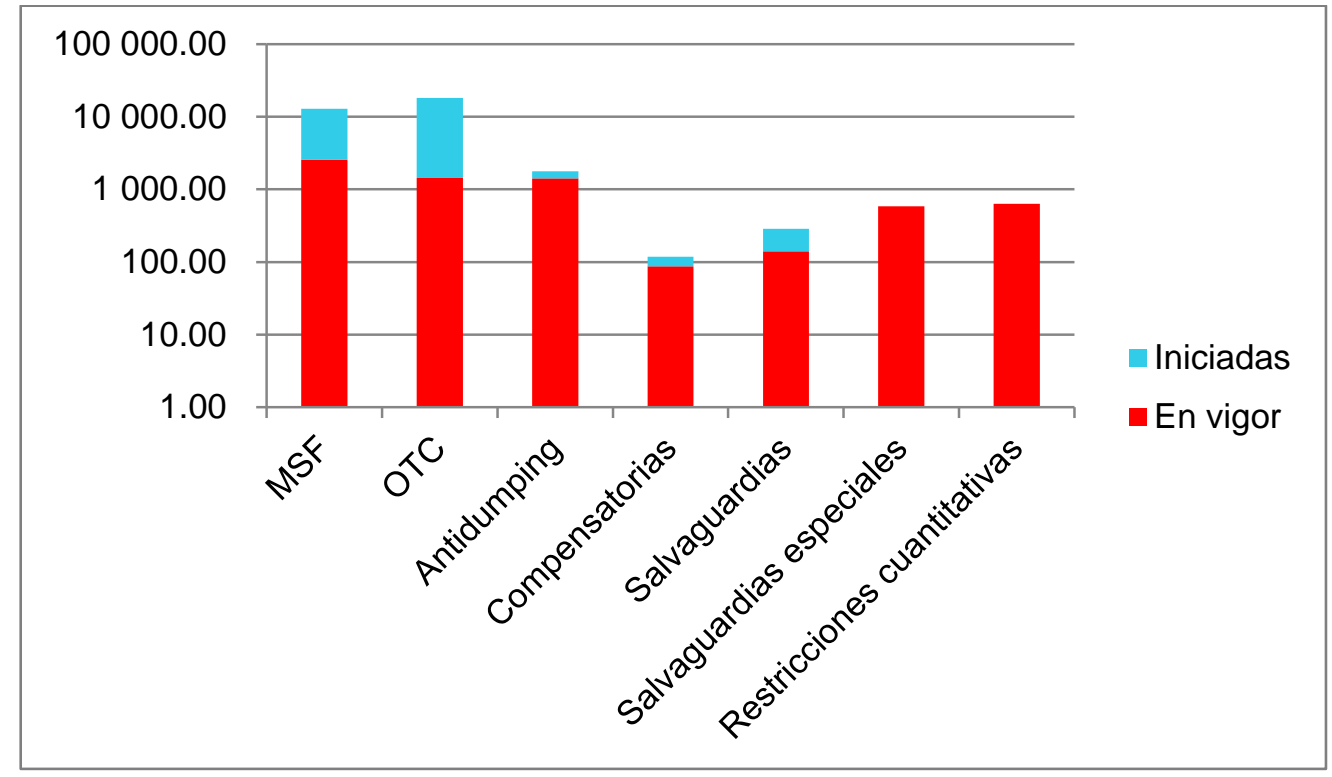

Fuente: Organización Mundial del Comercio, 2014

Algunas de las barreras que se pueden originar al libre comercio por medio de estas normas son las siguientes:

Valverde Arévalo, M. A. (2015). Un nuevo reto para el comercio exterior peruano: Las medidas sanitarias y fitosanitarias. Sinergia e Innovación, 3(1), 1-22. 
- La falta de estandarización

- La demora para el acceso a mercados de productos frescos

- Medidas sin sustento científico

- Desigualdad para competir entre países

- Barreras a favor de la industria local

- Confusión a los potenciales exportadores

\section{Falta de estandarización}

El Acuerdo MSF de la OMC establece en el Artículo №3, relativo a la armonización de las normas, que para concentrar en el mayor grado posible las medidas adoptadas por cada país, éstas se deberían basar en normas, directrices o recomendaciones internacionales; sin embargo, al ser cada país soberano también tiene derecho a establecer normas que representen un mayor nivel de protección sanitaria o fitosanitaria siempre que tengan algún sustento científico.

Si bien estas normas deben tener algún sustento, en repetidas ocasiones se pueden presentar significativas diferencias entre la norma internacional y la aplicada por algún país.

Las disparidades entre países principalmente se presentan en los productos agrícolas pues éstos son susceptibles de ser atacados por plagas por lo que resulta necesario el uso de plaguicidas para controlarlas. No obstante, antes de su aplicación es necesario evaluar la toxicidad que éstos pueden representar para el serhumano, animalesdomésticos,organismosqueno sonplagas,altrabajadorde campoy al medio ambiente.

La evaluación de la toxicidad es realizada por el CODEX y actualizada constantemente, estableciendo una lista de sustancias activas que pueden ser encontradas en los alimentos. A estas sustancias se les conoce como los límites máximos permitidos de residuos plaguicidas (en adelante LMR's).

Si bien el CODEX se encarga de la investigación necesaria, muchos países y bloques económicos como Estados Unidos y la Unión Europea consideran los resultados obtenidos de forma referencial y prefieren que sus propias autoridades sanitarias sean las encargas de estas evaluaciones y establezcan sus propios LMR's.

En el caso de Estados Unidos, si no se tiene reconocido un límite máximo de algún residuo plaguicida, simplemente el límite es cero y cualquier otro residuo que se encuentre ocasiona que el producto sea susceptible de ser rechazado (Lamont, 2014). Igualmente, en la legislación de la 
Unión Europea si es que no se tiene ningún nivel establecido se considera que el límite máximo es $0.01 \mathrm{mg} / \mathrm{kg}$ (Reglamento (CE) №396/2005 del Parlamento Europeo, 2005).

Un ejemplo de estas diferencias es el caso del espárrago, ya que el CODEX establece LMR's para 16 ingredientes activos; mientras que Canadá establece para el mismo producto 31, Australia 19, México 13, Estados Unidos 66 y la Unión Europea 440.

Figura 2 Número de sustancias activas que se regulan para la importación de espárrago fresco según destino

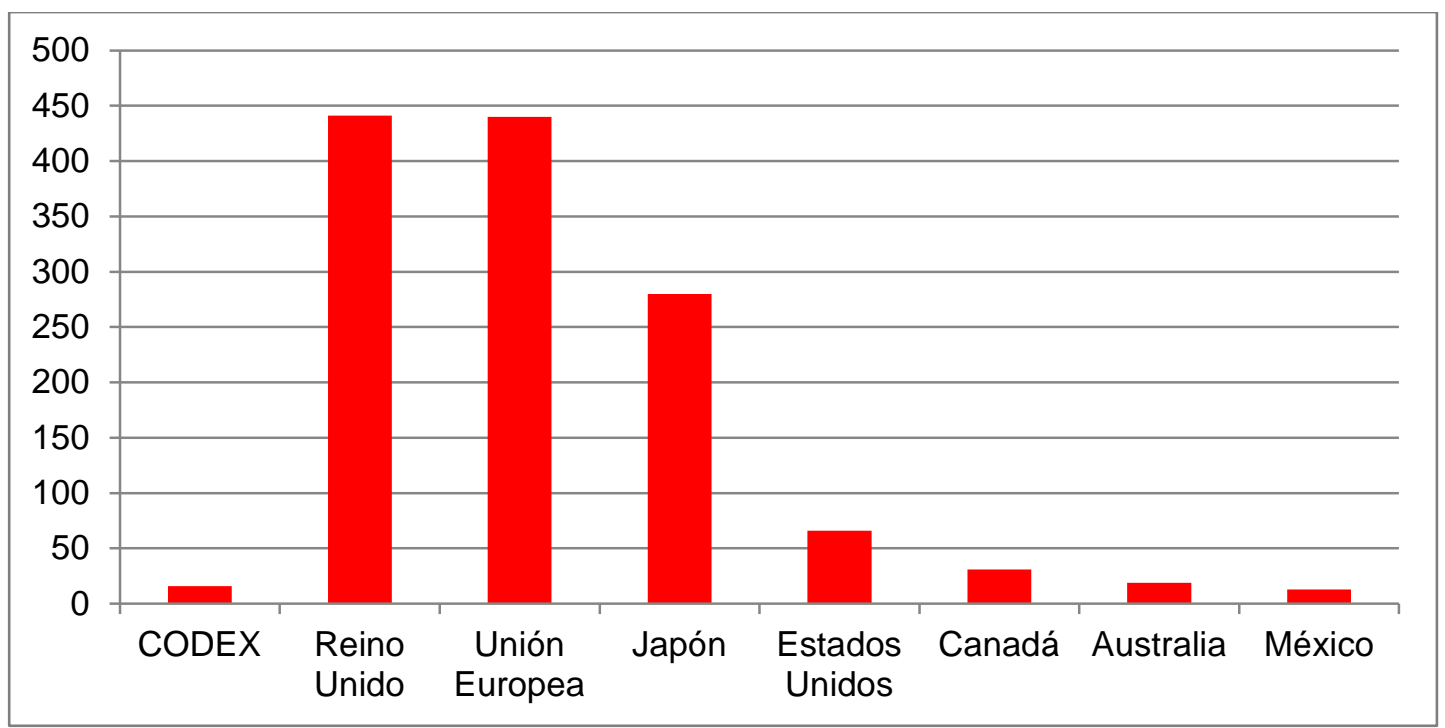

Elaboración propia a partir de datos obtenidos de Promperú

El caso del mango resulta similar, el CODEX establece límites máximos permitidos de residuos plaguicidas a 16 ingredientes activos mientras que Reino Unido 442. 
Figura 3 Número de sustancias activas que se regulan para la importación de mango fresco según destino

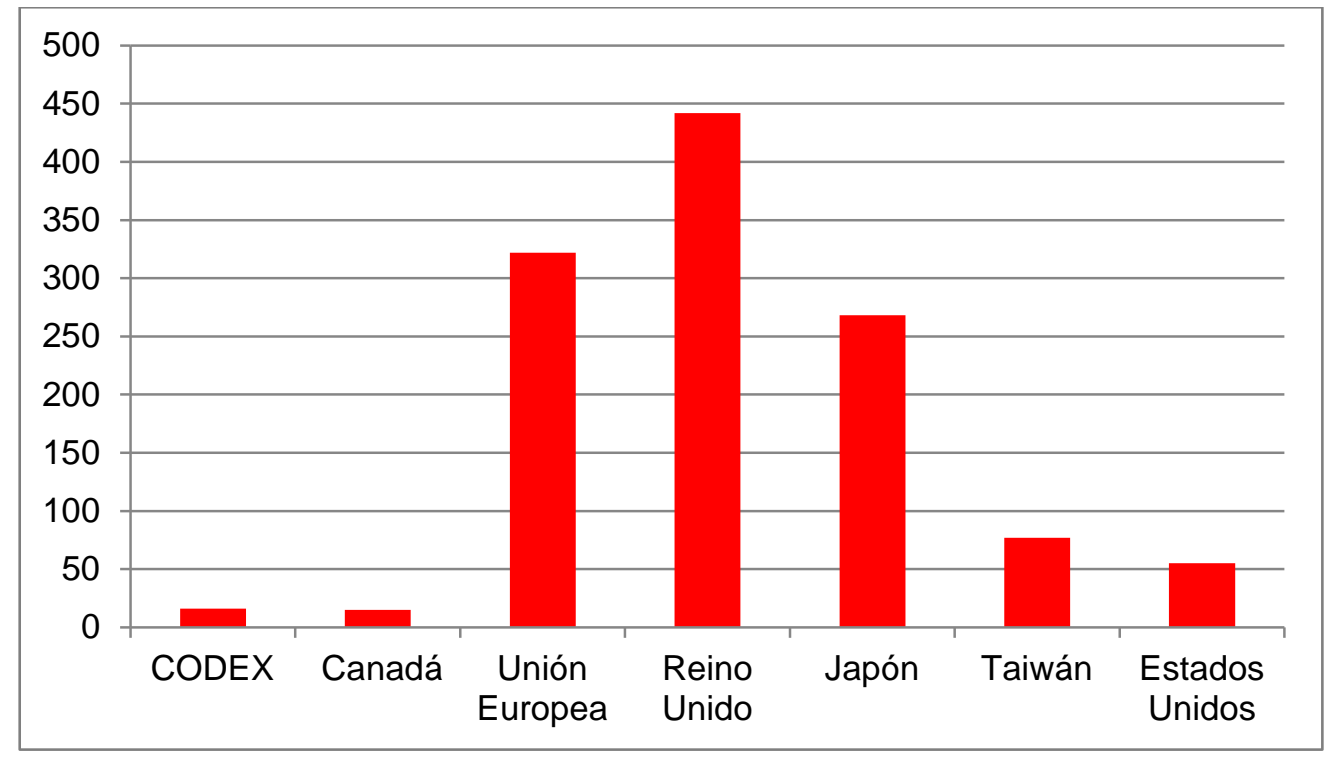

Elaboración propia a partir de datos obtenidos de Promperú

En este último caso, Reino Unido a pesar de ser parte de la Unión Europea tiene un listado mayor. Esta misma situación se puede presentar en el resto de países miembros al igual que en los Estados Unidos, los límites pueden variar de un Estado a otro (Comisión de Promoción del Perú para la Exportación y el Turismo, 2014).

Para la quinua, la Agencia de Protección Medioambiental de los Estados Unidos (EPA) ha establecido como único ingrediente activo permitido al glifosato con un valor de 5 ppm (Comisión de Promoción del Perú para la Exportación y el Turismo, 2014). Adicionalmente se cuenta con 28 ingredientes activos permitidos para su aplicación en etapas postcosecha, como el almacenamiento y transporte. Por el contrario la Unión Europea presenta una lista de 456 ingredientes activos.

En caso se desee ampliar la lista de LMR's para la quinua en el mercado norteamericano, el país o las empresas deberían financiar el estudio que equivale entre 200 mil a 300 mil dólares por cada nuevo componente y un tiempo promedio de casi 4 años hasta que sea aprobado (Lamont, 2014). Los estudios tendrían que ser financiados por la parte peruana en vista de que la quinua no se encuentra como producto priorizado en los Estados Unidos y no se tienen fondos destinados para su estudio.

Asimismo, existe la posibilidad que las partes por millón de un ingrediente activo puedan variar en grandes proporciones de un mercado a otro, por ejemplo el CODEX establece para las paltas un 
límite máximo de carbaryl de 15 ppm mientras que la Unión Europea lo sitúa en 0.5 ppm, Reino Unido en 0.01 ppm y Estados Unidos no lo tiene incluido por lo que el límite automáticamente es 0 ppm.

\section{Demora para el acceso a mercados}

En el Artículo $\mathrm{N}^{\circ} 4$ relativo a la equivalencia, se establece que un miembro aceptará como equivalentes las medidas sanitarias o fitosanitarias de otro miembro siempre que el miembro exportador pueda demostrar objetivamente al miembro importador que sus medidas logran el nivel adecuado de protección que establece este último. No obstante, para poder obtener la equivalencia pueden transcurrir varios años debido a la realización de los estudios necesarios y los trámites que se tienen que realizar ante la autoridad sanitaria del país importador.

Productos peruanos con exportaciones a mercados importantes aún tienen prohibido el ingreso a otros.

\section{Uvas frescas a Japón}

Uno de los casos que actualmente se encuentra en negociación es el de la uva fresca. El Perú es el séptimo exportador mundial de uvas ("Minagri: El Perú”, 2014) con más de 442 millones dólares en exportaciones en 2013. La uva peruana se exporta a mercados sumamente exigentes en temas fitosanitarios como la Unión Europea, Canadá y Estados Unidos.

Figura 4 Exportaciones peruanas de uva fresca, FOB USD (1994-2013)

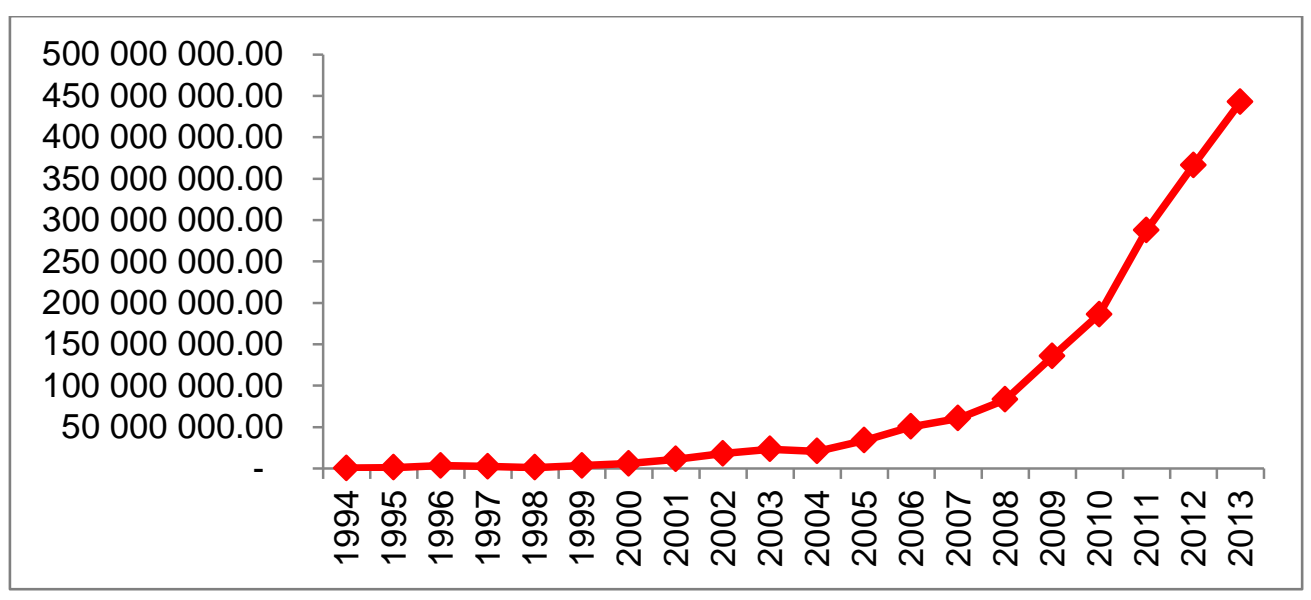

Elaboración propia a partir de datos obtenidos de Promperú

Valverde Arévalo, M. A. (2015). Un nuevo reto para el comercio exterior peruano: Las medidas 
Pese a este posicionamiento a nivel mundial de la uva nacional, no se tiene ninguna exportación a Japón. Esto se debe a que la autoridad sanitaria japonesa, el Ministerio de Agricultura Forestal y Pesca del Japón (MAFF, por sus siglas en inglés) aún no autoriza el ingreso de la uva peruana a ese mercado. Se han mandado cartas con los resultados de ensayos realizados a diferentes temperaturas y la mortandad de la mosca de la fruta, las mismas que han sido respondidas luego de casi un año y medio. No se cuenta con una fecha exacta para el ingreso de este producto al mercado japonés.

Hay que destacar, que entre junio y octubre ingresa al mercado japonés la uva local que tiene un 91\% del mercado (ProChile, 2012, p. 10).

\section{Palta Hass a Japón ${ }^{4}$}

Al igual que con las uvas, la palta continúa en la lista de productos prohibidos para la importación en Japón debido a que aún se presenta como sensible a la plaga de la mosca de la fruta. El Perú es el segundo exportador de paltas a nivel mundial con 12\% del total del mercado mundial ("Perú es el segundo", 2014). Asimismo las exportaciones de palta fresca a inicios de diciembre de 2014 ascienden a más de 300 millones de dólares (Comisión de Promoción del Perú para la Exportación y el Turismo, 2014).

Figura 5 Exportaciones peruanas de palta fresca, FOB USD (1994-2013)

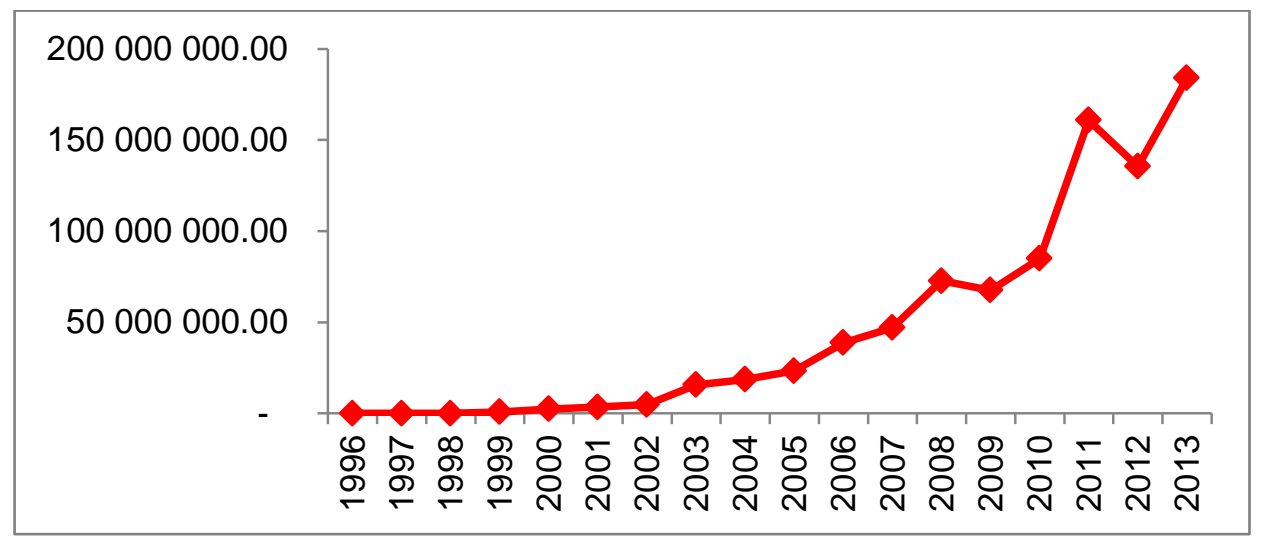

Elaboración propia a partir de datos obtenidos de Promperú

Un estudio presentado en 2012 por la Animal and Plant Health Inspection Service (APHIS) del Departamento de Agricultura de Estados Unidos, demuestra que:

\footnotetext{
${ }^{4}$ El fruto del árbol Persea americana conocido como "palta" en Perú es conocido como "aguacate" en otros países hispanohablantes.

Valverde Arévalo, M. A. (2015). Un nuevo reto para el comercio exterior peruano: Las medidas 
- La palta Hass con madurez comercial de Perú no es hospedante adecuado de A. Striata, A. Fraterculus, y C. Capitata.

- La palta Hass peruana no es una vía de ingreso para A. Striata

- La A. Striata no es una plaga de palta Hass en el país

- La palta Hass con madurez comercial es un no-hospedante condicional de A. Fraterculus y C. Capitata

SENASA afirma que ya los protocolos han sido concluidos y la palta ingresará a mercado japonés en 2015 (Torres \& Cruz, 2014).

Cabe resaltar que la palta Hass peruana podrá entrar al mercado japonés luego de 3 años de la entrada en vigencia del Acuerdo de Asociación Económica entre el Perú y Japón.

\section{Cítricos a Japón y otras economías}

Al igual que con la uva y la palta para el mercado japonés, se han realizado diferentes investigaciones para la aprobación de la mandarina a este mercado que aún se encuentran en evaluación por el MAFF.

Este producto ingresó a los Estados Unidos en 2006, cuando la APHIS, publicó la norma final que autoriza el ingreso de cítricos frescos del Perú a ese mercado (Servicio Nacional de Sanidad Agraria, 2010, p. 5). El TLC con este país recién entró en vigencia en 2009.

En 2008, la Administración General de Supervisión de Calidad, Inspección y Cuarentena de la República Popular China (AQSIQ) y el SENASA, aprobaron el Protocolo en el cual se establecen los requisitos fitosanitarios para laexportación de cítricos peruanos a China (Servicio Nacional de Sanidad Agraria, 2010, p. 5).

\section{Palta Hass a Estados Unidos}

La palta Hass ingresó a los Estados Unidos en el año 2010 después de años de tener preferencias unilaterales como el ATPA y el ATPDEA (Ministerio de Comercio Exterior y Turismo del Perú, s.f.).

Actualmente sólo se permite el ingreso de la variedad Hass y no de la Fuerte. Para su ingreso a este mercado es necesario presentar un certificado fitosanitario emitido por SENASA. 


\section{Medidas sin sustento científico}

\section{Novel Foods}

En el Acuerdo MSF en el Artículo 2: Derechos y Obligaciones Básicas,se establece que los miembros deben asegurarse de que las medidas sanitarias y fitosanitarias deben estar basadas en principios científicos de modo que no se mantenga alguna sin testimonios científicos suficientes.

El reglamento UE N²58/97 (NFR) define los nuevos alimentos e ingredientes alimenticios y regula su acceso al mercado europeo, considerándose como alimentos nuevos a los que no hayan sido comercializados en una medida significativa para el consumo humano en algún Estado Miembro de la Comunidad Europea antes del 15 de Mayo 1997 (Comisión de Promoción del Perú para la Exportación y el Turismo, 2013). Cabe resaltar que la medida no hace referencia explícitamente a alimentos nuevos en el mundo sino sólo que sean nuevos para ese mercado. Esta medida atenta directamente contra los productos tradicionales de la biodiversidad peruana, por lo que en las reuniones del Comité MSF en OMC se ha solicitado repetidas veces el sustento de esta medida sin que la Unión Europea pueda dar una respuesta clara.

Para que un producto "nuevo" tenga acceso al mercado deben transcurrir al menos tres años posteriores a la solicitud y se debe realizar por presentación del producto y por empresa.

Esta medida se encuentra en revisión por parte de las autoridades europeas y se estipula que la Comisión Europea quiere acortar los plazos de autorización de los nuevos alimentos de tres a dos años en promedio (Comisión de Promoción del Perú para la Exportación y el Turismo, 2014).

Productos peruanos como el aceite de sacha inchi recientemente han podido ingresar a este mercado a pesar de ser comercializados en otras partes del mundo y sólo para un determinado número de empresas por lo que limita la competencia.

\section{Desigualdad para competir entre países}

\section{Espárrago verde}

El Perú exporta espárrago verde fresco a los Estados Unidos desde mediados de los años ochenta. 
A partir del año 2001, debido a que se detectó la presencia de una plaga específica - la Copitarsia (un lepidóptero/polilla) - la autoridad fitosanitaria norteamericana dispuso un tratamiento con bromuro de metilo para las embarcaciones peruanas de este producto.

Esta disposición afecta la calidad de vida en anaquel de los espárragos pues se tiene que romper la cadena de frío para la fumigación, reduciéndola en más de 30\%; además, el costo aumenta en US\$ 0.30 por cada caja de cinco kilos (Instituto Peruano de Espárragos y Hortalizas, 2014).

En 2010, APHIS determinó la necesidad de aumentar la dosis de bromuro de metilo pues alegaban que la fumigación no estaba siendo eficaz, pero no se llegó a aplicar.

Luego de años de exportación, el tratamiento cuarentenario sigue siendo "ineficaz"; asimismo, cabe considerar que esta disposición no es imponible para los espárragos mexicanos en las mismas condiciones, lo cual resta competitividad al producto peruano (Instituto Peruano de Espárragos y Hortalizas, 2014).

\section{Barreras a favor de la industria local}

\section{Palta peruana versus manzanas chilenas}

En 2013, tanto Chile como Perú impusieron medidas fitosanitarias para la exportación de manzanas y palta respectivamente.

Chile impuso un nuevo protocolo a las paltas peruanas de exportación aduciendo que era debido a la plaga Avocado Sunblotch Viroid (ASBV) y a la mosca de la fruta; sin embargo, México como Estados Unidos tienen el Sunblotch en sus cultivos de palto, pero Chile nunca les puso barreras a estos países cuando se aprobaron las normas de ingreso (Barja Marquina, 2013). Además ya APHIS en 2012 había probado que la palta Hass peruana no era portador de la mosca de la fruta.

Prohass aducía que esta medida se había impuesto pues productores chilenos de palta no querían que los peruanos exporten a su mercado en el período de mayor demanda ("AGAP: Restricción chilena", 2013).

SENASA, en vista de esta situación, estableció una suspensión temporal a las importaciones de manzanas chilenaspor el inminente riesgo de que contengan BrevipalpusChilensis, ProeuliaAuraria, ProeuliaChrysopteris y Grapholita Molesta (Agencia Peruana de Noticias, 2013).

Ambos países negociaron y las autoridades desbloquearon los mercados.

Valverde Arévalo, M. A. (2015). Un nuevo reto para el comercio exterior peruano: Las medidas sanitarias y fitosanitarias. Sinergia e Innovación, 3(1), 1-22. 
Cabe resaltar que Chile sólo permite la entrada de palta de cinco de los diez departamentos con oferta exportable.

Figura 6 Mapa de departamentos con oferta exportable de palta

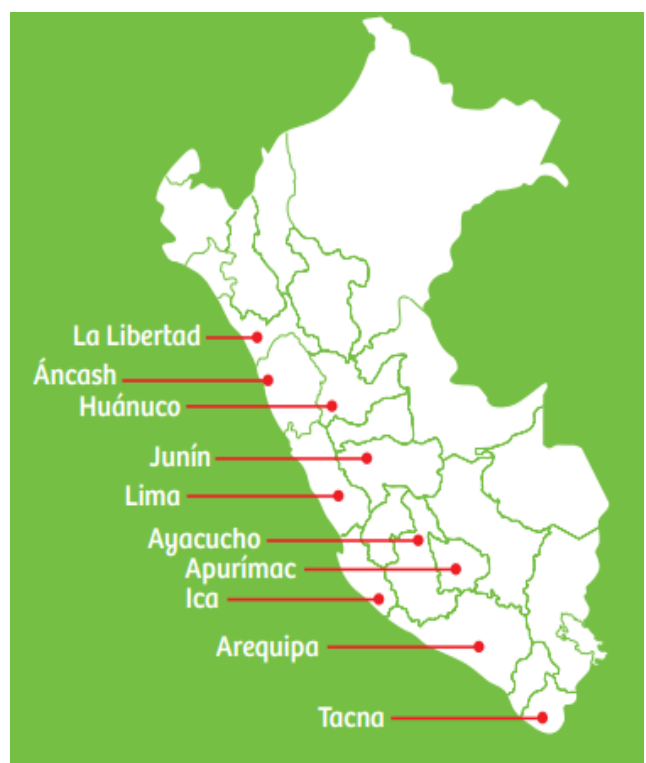

Fuente: Comisión de Promoción del Perú para las Exportaciones y el Turismo, 2011.

\section{Autorizados por Chile:}

- Sólo frutos variedad Hass provenientes de los departamentos de Ica, Lima, La Libertad, Moquegua, Tacna y Arequipa

- Sólo frutos variedad Fuerte provenientes de los departamentos de Arequipa, Moquegua y Tacna.

\section{Departamentos no reconocidos:}

- Ancash

- Huánuco

- Junín

- Ayacucho

- Apurímac

\section{Confusión a los potenciales exportadores}

Una primera fuente de referencia para los empresarios es la información estadística comercial de cada país; no obstante, el Perú exporta mermelada de papaya o de otros productos con 
combinaciones de papaya a los Estados Unidos, pero la papaya no tiene permitido el ingreso a este país.

Esto se debe a que la mayoría de restricciones se dan a los productos frescos; sin embargo, cuando éstos pasan por procesos químicos durante su industrialización es posible que los contaminantes y los residuos plaguicidas desaparezcan o disminuyan por lo que el producto ingresaría sin ningún problema al mercado extranjero.

La pulpa de mango que se exporta está elaborada de mangos de tercera calidad.De igual forma, se puede exportar pulpa de mango de un mango fresco que sobrepasa los LMR's o los contaminantes permitidos.

Por otro lado, es posible encontrar estadísticas de exportación de mandarina fresca a los Estados Unidos en los años 90 cuando se autorizó su entrada en la década del 2000. Esto se debe a que la FDA no se da abasto para controlar todos los embarques y menos del $0.2 \%$ son inspeccionados, siendo esa la razón de las estadísticas que se pueden encontrar en SUNAT, Infotrade, Siicex, entre otros (Lamont, 2014).

Asimismo, empresas peruanas exportan atún de aleta amarilla a los Estados Unidos a pesar de que el país tiene un embargo desde el 2002.

El embargo que se le ha dado al Perú en el tema de atún de aleta amarilla responde a una política de protección a los mamíferos acuáticos, como es el caso de los delfines, ya que por la modalidad de pesca del atún en el Pacifico puede afectar a la población de delfines que habitan en áreas cercanas a los cardúmenes de atún.

Sin embargo, las empresas que exportan han solicitado autorización a los Estados Unidos y han demostrado que sus actividades de pesca no afectan a los delfines, lo que se denomina como dolphin safe.

Igualmente, la quinua para los peruanos es un cereal, pero para los Estados Unidos no.

Al ser un producto nuevo para el mercado destino, su clasificación puede resultar distinta a la habitual en el mercado de origen, lo cual puede implicar más o menos medidas fitosanitarias a tener en cuenta para los exportadores. 
Finalmente, el camucamu ingresa a la Unión Europea como suplemento alimenticio pero tiene prohibida su entrada en otras presentaciones por lo que sería considerado como un alimento "nuevo".

\section{Malas prácticas empresariales y las MSF}

Si bien algunas medidas son bastante restrictivas para el comercio, éstas deben ser respetadas mientras se encuentren en vigencia de lo contrario se pueden establecer medidas aún más difíciles de cumplir o se puede cerrar el mercado de exportación. Un caso controversial que está sucediendo es de la quinua peruana de exportación.

\section{Quinua peruana con plaguicidas}

El Perú es uno de los principales países productores de quinua a nivel mundial, junto con Bolivia y Ecuador. Este producto, básicamente, se produce en zonas altoandinas y da trabajo a más de 70 mil pequeños y medianos agricultores, los cuales aún trabajan individualmente, pero se encuentran en proceso de asociarse (von Hesse La Serna, s.f.).

Las exportaciones de este producto, cada año, han ido aumentando significativamente, pues han pasado de casi 600 mil kilogramos exportados en 2005 a más de 19 millones en 2013. Además, esto representa un incremento de más de 80 millones de dólares exportados respecto a los mismos periodos.

Actualmente, la quinua se comercializa en más de sesenta mercados, siendo el principal los Estados Unidos a donde se exporta más de la mitad del total de exportaciones peruanas de quinua. En 2013, este país importó en total más de 44 millones de dólares de este producto, monto que casi duplicó los más de 22 millones importados en 2012 (von Hesse La Serna, s.f.).

Tabla 1Exportaciones de quinua en general

\begin{tabular}{|c|c|c|c|c|c|}
\hline Año & Valor FOB US\$ & $\begin{array}{c}\text { Volumen Bruto } \\
\mathbf{K g}\end{array}$ & $\begin{array}{c}\text { Valor } \\
\text { Unitario } \\
\text { Promedio } \\
\text { US\$/Kg }\end{array}$ & $\begin{array}{c}\text { Variación } \\
\% \text { Valor } \\
\text { FOB }\end{array}$ & $\begin{array}{c}\text { Variación } \\
\% \text { Volumen } \\
\text { Bruto Kg }\end{array}$ \\
\hline 2005 & $735,641.08$ & $595,389.20$ & 1.24 & & \\
\hline 2006 & $1,588,194.34$ & $1,305,938.07$ & 1.22 & 115.89 & 119.34 \\
\hline 2007 & $2,039,269.66$ & $1,577,702.11$ & 1.29 & 28.40 & 20.81 \\
\hline 2008 & $5,291,973.59$ & $2,186,865.59$ & 2.42 & 159.50 & 38.61 \\
\hline 2009 & $7,544,526.79$ & $2,832,098.30$ & 2.66 & 42.57 & 29.50 \\
\hline
\end{tabular}

Valverde Arévalo, M. A. (2015). Un nuevo reto para el comercio exterior peruano: Las medidas sanitarias y fitosanitarias. Sinergia e Innovación, 3(1), 1-22. 


\begin{tabular}{|c|c|c|c|c|c|}
\hline 2010 & $13,419,255.03$ & $4,938,727.87$ & 2.72 & 77.87 & 74.38 \\
\hline 2011 & $25,700,923.50$ & $8,224,588.60$ & 3.12 & 91.52 & 66.53 \\
\hline 2012 & $34,162,751.79$ & $11,876,280.48$ & 2.88 & 32.92 & 44.40 \\
\hline 2013 & $81,402,066.19$ & $19,382,030.60$ & 4.20 & 138.28 & 63.20 \\
\hline $2014^{5}$ & $93,061,994.36$ & $16,838,563.98$ & 5.53 & 14.32 & -13.12 \\
\hline & $\mathbf{2 6 4 , 9 4 6 , 5 9 6 . 3 3}$ & $\mathbf{6 9 , 7 5 8 , 1 8 4 . 8 0}$ & $\mathbf{3 . 8 0}$ & & \\
\hline
\end{tabular}

Elaboración propia a partir de datos obtenidos de Promperú

Este boom de la quinua ha originado que malos empresarios hayan mezclado quinua orgánica con quinua convencional que superaba los LMR's establecidos para abastecer a toda la demanda que se tiene de este producto.

La consecuencia de esta "estrategia" es el rechazo de numerosos envíos por parte de la FDA desde inicios del 2014.

Tabla 2Reporte de rechazos de exportaciones de quinua procedentes del Perú

\begin{tabular}{|l|l|l|}
\hline \multicolumn{1}{|c|}{ Fecha } & \multicolumn{1}{|c|}{ Producto } & \multicolumn{1}{c|}{ Motivo de rechazo } \\
\hline $18 / 11 / 2014$ & Quinua & Pesticidas \\
\hline $14 / 11 / 2014$ & Quinua & Pesticidas \\
\hline $30 / 10 / 2014$ & Quinua & Pesticidas \\
\hline $04 / 09 / 2014$ & Quinua & Sustancia nociva \\
\hline $22 / 08 / 2014$ & Quinua & Pesticidas \\
\hline $27 / 06 / 2014$ & Quinua & Pesticidas \\
\hline $02 / 06 / 2014$ & Quinua & Pesticidas \\
\hline $15 / 05 / 2014$ & Quinua & Pesticidas \\
\hline $24 / 04 / 2014$ & Quinua & Sustancia nociva \\
\hline $02 / 04 / 2014$ & Quinua & Pesticidas \\
\hline $14 / 02 / 2014$ & Quinua & Pesticidas \\
\hline
\end{tabular}

Elaboración propia a partir de datos obtenidos de la FDA

${ }^{5}$ Datos a Julio de 2014, sujetos a variación

Valverde Arévalo, M. A. (2015). Un nuevo reto para el comercio exterior peruano: Las medidas sanitarias y fitosanitarias. Sinergia e Innovación, 3(1), 1-22. 
Si las exportaciones de este producto continúan siendo detenidas es probable que se establezcan nuevas medidas fitosanitarias más exigentes, lo cual aumentaría los costos de exportación frente a la quinua de Bolivia que no tiene ningún rechazo.

\section{Conclusiones}

- Las medidas sanitarias y fitosanitarias conjuntamente con los obstáculos técnicos al comercio han devenido en las principales medidas restrictivas utilizadas por los países.

- A pesar de tener casi diez años de la entrada en vigencia del Acuerdo sobre MSF de la OMC no se ha logrado una verdadera armonización de las normas.

- Los países en desarrollo como el Perú son sumamente susceptibles a las medidas aplicadas por los países desarrollados por ser dependientes de las exportaciones de productos frescos.

- A pesar de tener sustento científico para demostrar la inocuidad de los alimentos, el reconocimiento de la equivalencia depende de otros factores externos al científico.

- El exportar un producto a un país desarrollado no asegura de que el mismo exportador pueda ingresar sin problemas a otro país.

- La entrada en vigencia de algún acuerdo comercial no necesariamente implica el ingreso de todos los productos frescos al mercado destino.

- En algunos productos aún se compite con desigualdad de condiciones.

- Para exportar es necesario revisar toda la legislación posible y actualizaciones además de consultar a las autoridades sanitarias nacionales respectivas.

- Las malas prácticas empresariales en temas sanitarios o fitosanitarios pueden ocasionar daños considerables a toda la industria de un país.

- La coordinación entre organismos estatales, gremios privados y la universidad debe ser constante para detectar cualquier posible riesgo y abrir más mercados para las agro exportaciones peruanas. 


\section{Referencias}

Agencia Peruana de Noticias. (2013, 17 de Diciembre). Perú y Chile firman acuerdo para ingreso de palta Hass. América Economía. Recuperado de http://www.americaeconomia.com/node/107170.

Alvarez, J. (s.f.). El GATT: Antecedentes y Propósitos. Recuperado de http://www.juridicas.unam.mx/publica/librev/rev/jurid/cont/11/pr/pr5.pdf.

Comisión de Promoción del Perú para las Exportaciones y el Turismo. (2011). Perú: Productos agrícolas. Recuperado de:

http://www.siicex.gob.pe/siicex/resources/sectoresproductivos/Catalogo\%20Agro.pdf.

Comisión de Promoción del Perú para la Exportación y el Turismo. (2013). Regulación Novel Food. Recuperado de http://www.siicex.gob.pe/siicex/resources/calidad/171612013radB94C7.pdf

Comisión de Promoción del Perú para la Exportación y el Turismo. (2014). Guía Referencial de Límites de Máximos de Residuos de Plaguicidas Químicos de Uso Agrícola (P.Q.U.A:) para los Principales Productos Hortofrutícolas de Exportación Según Mercados de Destino. Recuperado dehttp://export.promperu.gob.pe/calidad/.

Comisión de Promoción del Perú para la Exportación y el Turismo. (2014). InfoTrade [página web]. Recuperado dehttp://infotrade.promperu.gob.pe/index.asp

Comisión de Promoción del Perú para la Exportación y el Turismo. (2014). U.E. Un nuevo reglamento para fomentar el innovación en el sector alimentario a través de los: Nuevos Alimentos. Recuperado de:

http://www.siicex.gob.pe/siicex/portal5ES.asp? page $=726.72400 \&$ portletid $=$ aim wlistaler ta\&scriptdo=usp aim wlistdalerta\&pc alerta $=21951$

Convención Internacional de Protección Fitosanitaria. (2014). Historia de la CIPF [página web]. Recuperado de https://www.ippc.int/es/history-of-the-ippc/ . 
Barja Marquina, R. (2013). Prohass irá hasta la OMC si Chile sigue bloqueando a palta Hass. Recuperado de http://gestion.pe/economia/prohass-ira-hasta-omc-si-chile-sigue-bloqueandopalta-hass-2076103

AGAP: Restricción chilena a la palta peruana es "proteccionismo sin fundamento". (2013, 16 de Setiembre). Recuperado de http://gestion.pe/economia/agap-restriccion-chilena-paltaperuana-proteccionismo-sin-fundamento-2076247.

Minagri: El Perú es el sétimo exportador mundial de uva. (2014). Recuperado de http://gestion.pe/economia/minagri-peru-setimo-exportador-mundial-uva-2110883

Perú es el segundo exportador de palta del mundo con el 12\% del total. (2014) Recuperado de http://gestion.pe/economia/peru-segundo-exportador-palta-mundo-12-total-2109869

Instituto Peruano de Espárragos y Hortalizas. (2014). [Ayuda Memoria Espárrago Verde Peruano y el Mercado de EE. UU.] Datos no publicados.

Lamont, M. (2014). Situación actual para la exportación de quinua a Estados Unidos. Actualización de los requisitos y soluciones. Desayuno-Conferencia: "Exportación de quinua a Estados Unidos: Actualizando los requisitos" Cámara de Comercio Americana del Perú, Lima.

Ministerio de Comercio Exterior y Turismo. (2014). Japón. Recuperado de:

http://www.mincetur.gob.pe/newweb/Portals/0/documentos/ASIA/TOKIO.pdf

Ministerio de Comercio Exterior y Turismo. (s.f.). Guía práctica para los Estados Unidos.

Recuperado de http://www.mincetur.gob.pe/newweb/Portals/0/comercio/guiaexportacion/pdfs/12 ESTADOS UNIDOS.pdf

Organización Mundial de Sanidad Animal. (2007). Reconocimiento por la OIE del estatus sanitario de países. Recuperado de http://www.oie.int/fileadmin/Home/esp/Publications \%26 Documentation/docs/pdf/bulletin/B ull 2007-1-ESP.pdf

Organización Mundial de Sanidad Animal. (2011). Erradicación de la peste bovina. Recuperado de http://www.oie.int/fileadmin/Home/esp/Publications \%26 Documentation/docs/pdf/bulletin/B ull 2011-2-ESP.pdf 
Organización Mundial del Comercio. (2012). Medidas Sanitarias y Fitosanitarias. Recuperado de http://ecampus.wto.org/admin/files/Course 523/CourseContents/SPS-R3-S-Print.pdf

Organización Mundial del Comercio. (2013). Informe sobre el comercio mundial 2013: Factores que determinan el futuro del comercio. Recuperado de http://www.wto.org/spanish/res s/booksp s/wtr13-2b s.pdf

Organización Mundial del Comercio. (2014) IntegratedTradelntelligence Portal (I-TIP). Recuperado de https://i-tip.wto.org/goods/Forms/GraphView.aspx.

ProChile. (2012). Estudio de mercado de uva para el mercado Japón. Recuperado de http://www.prochile.gob.cl/wp-content/blogs.dir/1/files mf/documento 1206 12173416.pdf.

Reglamento (CE) №396/2005 del Parlamento Europeo y del Consejo relativo a los límites máximos de residuos de plaguicidas en alimentos y piensos de origen vegetal y animal y que modifica la Directiva 91/414/CEE del Consejo, 23 de febrero de 2005.

Servicio Nacional de Sanidad Agraria. (2010). Procedimiento: Certificación Fitosanitaria de Cítrico (Citrus spp) Destinadas a la Exportación. Recuperado de http://admin.senasa.gob.pe/RepositorioAPS/0/2/JER/EXPORTACION/PROCEDIMIENTOS\% 20PARA\%20LA\%20CERTIFICACION\%20FITOSANITARIA\%20DE\%20CITRICOS\%20DE\% 20EXPORTACION.pdf

Servicio Nacional de Sanidad Vegetal. (s.f.). Gestión en Exportaciones Peruanas: Mercados con Acceso Año 2010 y 2011. Obtenido de http://www.senasa.gob.pe/0/modulos/JER/JER Interna.aspx?ARE=0\&PFL=2\&JER=4205.

Torres, A., \& Cruz, J. (2014, 27 de Enero). Cítricos, Palta Hass y Uvas Peruanas Ingresarían a Japón en 2015. Recuperado de http://agraria.pe/noticias/citricos-palta-hass-y-uvasperuanas-ingresarian-japon-en-2015.

López García, J. L. \& Berga Monge, A. (2007). Unidad Didáctica 1 La Organización de la Seguridad Alimentaria. Recuperado de http://ocw.upm.es/tecnologia-dealimentos/seguridad-alimentaria/contenidos/Lecciones-y-Test/Lec-1.1..pdf.

von Hesse La Serna, M. (s.f.). La Quinua como Oportunidad . Obtenido de http://www.minag.gob.pe/portal/download/pdf/especiales/granos-andinos/exposicionquinua.pdf. 